\title{
Quand le Malin fait de l'esprit. Le rire au Moyen Âge vu depuis l'hagiographie
}

In: Annales. Histoire, Sciences Sociales. 52e année, N. 3, 1997. pp. 457-475.

\author{
Abstract \\ Witty Devil: Laughter in the Middle Ages as seen from Hagiology.L. Moulinier.
}

As a passion of the soul expressing itself loudly through the body, laughter was generally held in great suspicion by the medieval Church, especially in monastic life, where it was considered as the enemy of silence and seriousness.

Comical elements in edifying texts, telling of the life of an abbot and an abbess in the perspective of their canonizations, are all the more surprising. This study intends to analyze the function of such episods in hagiographic narrative (on the basis of two twelfth-century Vitae, on Bernard de Clairvaux and Hildegard of Bingen) and the mechanism and scope of a certain type of laughter in the Middle Ages.

Citer ce document / Cite this document :

Moulinier Laurence. Quand le Malin fait de l'esprit. Le rire au Moyen Âge vu depuis l'hagiographie. In: Annales. Histoire, Sciences Sociales. 52e année, N. 3, 1997. pp. 457-475.

doi : 10.3406/ahess.1997.279580

http://www.persee.fr/web/revues/home/prescript/article/ahess_0395-2649_1997_num_52_3_279580 


\section{QUAND LE MALIN FAIT DE L'ESPRIT Le rire au Moyen Age vu depuis l'hagiographie}

Laurence MOULINIER

Aux prises avec la question du rire au Moyen Age, labile comme le sourire du chat du Cheshire, nous avons choisi de le traquer là où on l'attend a priori le moins, dans les récits hagiographiques et en particulier dans deux Vies du $12^{\mathrm{e}}$ siècle, où un même comique s'exerce aux dépens du saint, Bernard de Clairvaux (1090-1153) dans un cas et Hildegarde de Bingen (1098-1179) dans l'autre.

Le maître d'œuvre de la Vita prima sancti Bernardi est Geoffroy d'Auxerre, qui avait été son secrétaire et qui prit dès 1145 , en raison sans doute de la mauvaise santé de l'abbé, l'initiative de faire écrire sa Vie' : Guillaume de Saint-Thierry s'attela, de 1146 à sa mort en 1148, à la rédaction du livre premier, et le second, dans lequel prend place notre document, a pour auteur Arnaud (ou Ernaud), abbé de Bonneval ; écrit entre 1148 et 1153, il englobe les années 1130-1145 de l'existence de Bernard. A Geoffroy échut le soin de raconter, en trois livres, les dernières années puis, après l'échec d'une première tentative de canonisation en 1163 , d'opérer une révision de l'ensemble de la Vita.

La Vita sancta Hildegardis mêle plusieurs voix et est due principalement à deux auteurs : Godefridus, moine du Disibodenberg, en aurait entamé la rédaction du vivant même de l'abbesse, mais la mort (vers 1181) l'empêcha d'aller au-delà du liber primus de gestis sancta ; Theodoricus, moine d'Echternach, acheva son œuvre vers 1187, dotant la Vita d'un prologue et de deux autres livres, le liber secundus de visionibus sancta et le liber tertius

Je tiens à remercier Sofia Boesch Gajano, Piero Brogi et Antonella Romano pour l'aide qu'ils m'ont apportée dans la mise au point de cet article, lors du séjour d'études que j'ai fait à Rome grâce à une bourse Lavoisier du ministère des Affaires étrangères en 1995.

1. Pour plus de détails voir A. H. BREDERO, «La vie et la Vita prima», dans Bernard de Clairvaux. Histoire, mentalités, spiritualité. Colloque de Lyon-Citeaux-Dijon, Paris, Le Cerf, 1992, pp. 53-82.

Annales HSS, mai-juin 1997, $n^{\prime \prime} 3$, pp. 457-475. 


\section{LE RIRE}

de miraculis et morte beatce dans lequel figure l'épisode qui nous retiendra, et que l'on peut dater de 1167 environ. Cette Vita fut rédigée, elle aussi, dans l'espoir d'une canonisation de son hérö̈ne, mais, contrairement à Bernard qui fut canonisé en 1174, Hildegarde ne jouit jamais d'une reconnaissance officielle.

Les deux épisodes présentent d'évidentes similitudes. Dans les deux cas, il s'agit d'un miracle thaumaturgique opéré par le saint de son vivant - l'un à Milan, l'autre en Rhénanie -, ou plus précisément d'un exorcisme se déroulant en plusieurs étapes en raison de la résistance qu'oppose l'esprit malin: Bernard et Hildegarde sont tenus en échec et ridiculisés par un démon moqueur habitant le corps d'une femme, damoniaca. A quels mécanismes faisait appel le rire à l'œuvre dans ces textes et dans quel but, au nom de quelle conception du comique, un récit édifiant mettait-il en scène sous un jour défavorable le personnage dont il voulait prouver la perfection? Une telle entreprise n'était-elle pas somme toute à double tranchant? Pour répondre à ces questions, on ne se limitera pas au cadre étroit de ces deux textes : le rire, cette étonnante faculté de l'homme, ce phénomène mettant en jeu le physique et le mental, suscitait en effet au Moyen Age des attitudes, des discours et des jugements fort divers mais, on le verra avec Hildegarde, pas pour autant inconciliables.

\section{La femme et le démon}

Par le rôle qu'y joue l'élément féminin, ces récits confirment d'abord un phénomène mis en évidence par Pierre-André Sigal pour la France des $11^{\mathrm{c}}-12^{\mathrm{c}}$ siècles : si le pourcentage des miraculées dans les miracles in vita est toujours inférieur à celui des miracles posthumes, les femmes rencontrant sans doute de plus grandes difficultés pour se rendre aux sanctuaires ${ }^{2}$, en revanche «la seule catégorie où les femmes sont proportionnellement plus nombreuses dans les miracles in vita que dans les miracles posthumes est celle où les femmes ne viennent pas de leur plein gré ». Et de fait, dans nos deux Vita, la possédée n'est pas venue d'elle-même auprès du saint : la démoniaque guérie par Bernard lui a été amenée par son mari, et la femme libérée par l'action de Hildegarde a été conduite auprès d'elle par des amis et protecteurs 3 .

En ce qui concerne non plus le sexe du fidèle affligé mais celui du saint guérisseur, le plus remarquable est qu'un exorcisme soit pratiqué par une femme : les saints faisaient de leur vivant une assez grande place à cette pratique $^{4}$, et Bernard notamment a beaucoup agi dans la guérison des

2. P.-A. Sigal, L'homme et le miracle dans la France médiévale (XI'-XII siècle), Paris, Le Cerf, 1985, p. 259 : « Peut-être les saints étant surtout des hommes d'Église, et leur entourage essentiellement masculin, l'accès des femmes auprès d'eux était-il plus difficile ? ».

3. Vita sancta Hildegardis, M. KLAES éd., Turnhout, Brepols, 1993, p. 63 : amicis et tutoribus femince persuaserunt, ut ad beatam virginem eam perducerent.

4. P.-A. Sigial, L'homme et le miracle..., op. cit., p. 238. 
possédés ${ }^{5}$, mais le cas de Hildegarde est tout à fait rare pour l'époque. Certes, aux premiers siècles du christianisme, l'activité d'exorciste n'était pas spécifiquement masculine : «Jusque vers le milieu du $3^{\mathrm{e}}$ siècle, comme l'attestent Tertullien ou Minucius Felix, tous les fidèles pouvaient prétendre exorciser ${ }^{6}$. Au $12^{\mathrm{e}}$ siècle, en revanche, l'exorcisme est exclusivement de la compétence des hommes, et en entreprenant de libérer la possédée, Hildegarde s'arroge une prérogative masculine, comme lorsqu'elle se lance - à quatre reprises - dans des tournées de prédication devant le clergé et le peuple de différentes villes d'Allemagne. Débordant le cadre alors imparti aux femmes, elle ne craignit pas d'agir en homme, et aucune protestation ne paraît s'être élevée contre les libertés qu'elle prenait. Une telle impunité peut sans doute s'expliquer par la force de son caractère et de ses relations et surtout par le prestige et l'autorité dont elle jouissait depuis que le pape Eugène III, un cistercien ami de saint Bernard, avait officiellement reconnu son don prophétique lors du synode de Trèves en 1147-1148. Mais il y a plus: dans le texte qui nous intéresse ici, c'est à la demande du monde masculin, en l'occurrence l'abbé et les frères de Brauweiler, que Hildegarde se fera exorciste.

Sigewize, la possédée ( «obsédée » serait plus fidèle, mais est d'un emploi délicat en français) n'en était pas à sa première tentative de guérison : souffrant depuis huit ans, elle avait été amenée à Brauweiler dans l'espoir d'y être délivrée par les mérites de saint Nicolas, patron de cette communauté de bénédictins, mais son attente avait été déçue. Imploré par les moines, l'esprit malin fit savoir que seule une vieille femme habitant la Rhénanie supérieure, vetula in superioribus Rheni partibus, pourrait le chasser; on demanda donc à une femme, bien vivante quoique âgée, et jouissant alors seulement d'une réputation de sainteté, d'accomplir ce qu'un saint de sexe masculin, et connu de longue date pour ses vertus, n'avait pu réaliser post mortem. Autre point commun entre nos deux Vitae: Bernard aussi n'entre réellement en scène et en action qu'à partir du moment où le recours à un autre saint, saint Syr, s'est avéré inopérant ${ }^{7}$. En d'autres termes, si le miracle de guérison est ici une preuve de la sainteté de Bernard et de Hildegarde - les vertus ne supplanteront le miracle comme critère de sainteté qu'à partir du $13^{\mathrm{e}}$ siècle - , cette sainteté nouvelle se manifeste et revêt un caractère d'évidence par contraste, à la faveur d'une défaillance de saints « traditionnels».

5. Ses biographes en rapportent moult cas «prenant parfois l'allure de véritables cures analytiques " (J. BERLIOZ, "Saint Bernard dans la littérature satirique. De l'Ysengrimus aux Balivernes des courtisans de Gautier Map (XII"-XIII" siècles) », dans Vies et légendes de saint Bernard, Présence cistercienne, 1993, pp. 211-228, p. 221). Que Jacques Berlioz soit ici chaudement remercié de m'avoir communiqué ses travaux.

6. Cf. l'article «Exorciste» dans Dictionnaire de théologie catholique, commencé sous la direction d'A. Vacant et A. Mangenot, continué sous celle d'E. Amann, Paris, Letouzey et Ané, 1939, t. 5, $2^{\mathrm{c}}$ partie, col. 1780-1786.

7. "Mais l'homme de Dieu l'envoya à l'église de saint Syr. Saint Syr voulut le céder à son hôte et ne fit aucun bien à cette femme »; cf. Sancti Bernardi Vita prima, lib. II, Patrologie latine (désormais $P L$ ) 185, col. 280. 


\section{LE RIRE}

L'adhésion au miracle n'allait pas pour autant de soi, et il pouvait toujours se trouver des esprits forts pour contester le pouvoir thaumaturgique du saint ; aussi la littérature hagiographique ne perdit-elle jamais tout à fait son caractère de "littérature de combat ${ }^{8}$ sensible dans le présent extrait de la Vita Hildegardis: Theodoricus en reste le principal narrateur mais il mêle à son propre récit des passages supposément autobiographiques de l'abbesse (la voix de Hildegarde se fait donc entendre aussi), et en outre, trois lettres échangées par Hildegarde et l'abbé de Brauweiler au sujet de la "possédée » nous sont parvenues. Un même événement reçoit ainsi trois éclairages complémentaires et leurs différences sont significatives.

L'abbesse est d'abord sollicitée par écrit, après l'échec de l'invocation à saint Nicolas, et suite surtout à une requête exprimée par le démon luimême : selon Theodoricus, il aurait dit par la voix de la jeune possédée, que seule « une petite vieille» pourrait le déloger, se moquant d'elle au passage en déformant son nom, Hildegardis, en Scrumpilgardis ${ }^{9}$. Or l'ironie mordante prêtée à l'esprit malin a toutes les apparences d'être du cru de Theodoricus, si l'on en juge par la comparaison de ce passage avec le propre témoignage de Hildegarde et celui de l'abbé de Brauweiler: dans un des passages autobiographiques, l'abbesse rapporte que le démon l'a désignée par le terme de vetula, mais ne dit rien du sobriquet forgé par l'esprit malin sur son nom ${ }^{10}$; quant à l'abbé de Brauweiler qui se fait le porte-parole de ses moines, il ne fait aucune mention de ces deux appellations désobligeantes, et flatte au contraire Hildegarde pour s'attirer ses lumières et son secours ${ }^{\prime \prime}$.

Plus qu'une omission ou une réticence de la part de Hildegarde ou de son correspondant, ne faut-il pas voir dans ce décalage des sources la main de Theodoricus, désireux de prouver à tout prix la sainteté de son personnage et de lui attribuer des pouvoirs miraculeux auxquels elle n'a jamais personnellement prétendu? Il le dit clairement: le Tout-Puissant aurait pu aisément accorder le pouvoir de libérer l'obsessa à l'un des saints auprès desquels elle avait été conduite auparavant, mais il a voulu, en «transférant la gloire de ce miracle à la vierge sainte ", que soit révélé à tous et de son vivant l'éclat de ses mérites ${ }^{12}$. Et Theodoricus d'affirmer qu'elle avait entre autres dons celui de guérir les possédés, donnant à un épisode singulier

8. P.-A. Sigat, L'homme et le miracle..., op. cit., p. 215.

9. Vita sancta Hildegardis, op. cit., p. 58 : dixit se suum non relicturum vasculum, nisi per cujusdam vetula in superioribus Rheni partibus, sicut ipsa supra dixerat, consilium et auxilium, nomen ejus evertens, et quod Scrumpilgardis vocaretur, deridens.

10. Ibid., p. 57 : Cumque mulier illa per plurima loca ad sanctos esset deducta, spiritus qui eam oppresserat, devictus meritis sanctorum et votis populorum, vociferabatur, quod in superioribus Rheni partibus Vetula quadam esset, per cujus consilium expellendus foret.

11. Ibid., p. 59: Demon enim iste nunc quadam die conjuratus, tandem manifestavit nobis mulierem hanc obsessam liberandam esse per virtutem vestra contemplationis, magnitudinem divina revelationis, et p. 63 (deuxième lettre de l'abbé de Brauweiler) : Nobis autem iterum eum conjurantibus et insistentibus tandem respondit, quod vas possessum non nisi in presentia vestra relinquat.

12. Ibid., p. 63. 
valeur d'exemple ${ }^{13}:$ animé par ces intentions édifiantes, et conscient peutêtre qu'un exorcisme opéré par une femme n'a rien de très orthodoxe, l'hagiographe enrichit donc son récit de force détails, pour conclure à la réussite de l'action divine en Hildegarde, en ayant soin de préciser que celle-ci ne s'attribua en rien le mérite de la libération de Sigewize ; les propos ironiques de l'esprit malin auraient eux-mêmes subi une surenchère sous sa plume, afin de faire agir la moquerie comme un révélateur de plus, au sens photographique du terme, de la sainteté de Hildegarde.

Du récit placé dans sa bouche, il ressort qu'elle finit par voir la jeune femme et prêter son concours à sa libération, après un refus initial motivé par sa propre maladie puis une première réponse au problème par écrit. Dans cette lettre "dictée par l'Esprit saint », à lire à haute voix au-dessus du corps de la possédée, Hildegarde esquisse une typologie des démons : selon elle, l'esprit en question est de ceux qui séjournent volontiers en compagnie des hommes, et ne craignent guère la croix du Seigneur, les reliques, et autres instruments du service de Dieu. Au contraire, ils s'en moquent ${ }^{14}$ - et l'on retrouve ici le thème de la voie tracée par le Christ dans les airs, domaine des démons, présent par exemple chez saint Paul et glosé par différents Pères de l'Église : les démons qui habitent les airs essaient d'empêcher les âmes de monter au ciel, mais la voie vers le Ciel a été rouverte par le Christ en croix ${ }^{15}$.

Hildegarde proposait ensuite une complexe mise en scène pour chasser le démon: il s'agissait de désigner sept prêtres vertueux, en lieu et place d'Abel, Noé, Abraham, Melchisedech, Jacob, Aaron et du Christ lui-même ; à tour de rôle, une verge dans la main, ils devaient prononcer des paroles aptes à chasser l'esprit et représenter tous ensemble les sept dons de l'Esprit saint, car comme il avait plané sur les eaux et animé l'homme de son souffle, le Saint-Esprit devait expulser le démon ${ }^{16}$. Or Theodoricus, attaché à mettre en vedette la sainteté de l'abbesse, ne précise pas dans son récit si cette thérapie de choc, obéissant à un ordo très particulier et ne faisant intervenir que des hommes, a été ou non appliquée. D'après lui, un premier effet fut atteint à la lecture de la formule finale de ladite lettre, où la sagesse divine, par la bouche de la "pauvre et ignorante petite figure féminine » qu'est Hildegarde, enjoint au malin de quitter le corps qu'il obsède; dans un grand hurlement, le démon quitte alors son habitacle, son « vase », la

13. Ibid., p. 55 : Inter catera autem virtutum insignia, data est a Domino sancta virgini ab obsessis corporibus damones ejiciendi gratia, sicut de quadam nobili et adhuc tenera atatis femina.

14. Ibid., p. 60 : et deridet, nec multum pertimescit.

15. Sur ce motif, voir par exemple J. DaniÉlou, «Les démons de l'air dans la "Vie d'Antoine" », Studia Anselmiana, XXXVIII, 1956, pp. 136-147.

16. Cf. Vita sancta Hildegardis, op. cit., pp. 60-61; pour une analyse de cet exorcisme quasi théâtral, voir P. DronkE, «Problemata Hildegardiana », Mittellateinisches Jahrbuch, XVI, 1981, pp. 97-131; sur l'exorcisme, voir désormais aussi S. GouguenHEIM, « La sainte et les miracles. Guérisons et miracles d'Hildegarde de Bingen », Hagiographica, II, 1995, pp. 157176. 


\section{LE RIRE}

possédée paraît guérie et, notons-le, elle se prosterne devant l'autel de saint Nicolas ${ }^{17}$.

Mais la rechute ne tarde pas - le démon ne laisse en paix Sigewize qu'une petite heure, d'après les frères de Brauweiler ${ }^{18}$ - et c'est seulement lorsque la malheureuse est mise en présence de la sainte, malgré les appréhensions initiales de toute la communauté féminine du Rupertsberg, que la libération a lieu : sans intermédiaire cette fois, Hildegarde intime en nom propre à Satan d'abandonner le corps de la femme et de céder ainsi la place à l'Esprit saint ${ }^{19}$. Elle est obéie, 1'esprit immonde quitte enfin, par ses parties les plus intimes, le corps dont il s'était emparé, et Theodoricus peut affirmer, par la voix de l'abbesse, que la possédée ne subit plus nulle attaque du diable aussi longtemps qu'elle vécut ${ }^{20}$. Comme dans la Vie de saint Bernard, la libération finale est donc obtenue, mais le biographe de Hildegarde prend des précautions supplémentaires liées au sexe de sa candidate à la sainteté : le face à face entre l'abbesse et la possédée, c'est-à-dire l'exorcisme à proprement parler, est ici en quelque sorte différé, et en tout cas dûment justifié par le relatif échec du premier procédé recommandé par Hildegarde.

Celle-ci n'a rien à envier à Bernard quant aux résistances et aux avanies que lui fait subir le Malin (tous deux sont également moqués et atteints au plus profond de leur être, dans leur identité même), mais seule Hildegarde se voit offensée dans son sexe par ce démon qui fait de l'esprit à ses dépens. Lorsqu'il fait savoir à quelle condition il laissera en paix la possédée, il ne la désigne en effet pas par son nom mais par deux appellations destinées à la tourner en dérision : vetula, «petite vieille », et Scrumpilgardis, sobriquet que l'on pourrait rendre par «Ridegarde», mêlant en un jeu de mots l'adjectif signifiant « ridé », « ratatiné », schrumpelig en allemand, et un des deux radicaux du prénom de Hildegarde. Flétrie pour son âge - elle a près de 70 ans _ , elle est ravalée au même rang que la damoniaca libérée par saint Bernard, que son propre démon appelait canicula mea, «ma petite vieille», et le qualificatif de vetula qui lui est appliqué est riche de sens : il stigmatise à la fois les carences, notamment intellectuelles, considérées alors comme inhérentes au sexe féminin, et les tares de la senectus - comme le rappellent Jole Agrimi et Chiara Crisciani, une

17. Ibid., pp. 61-62: «Et ergo indocta et paupercula feminea forma, o blasphemice et derisor spiritus, tibi dico in illa veritate, qua ego paupercula et indocta forma de lumine sapientia hac vidi et audivi, et per eamdem sapientiam tibi pracipio, ut de ista homine in vera stabilitate, et non in turbine tuce instabilitatis exeas", idem nequam spiritus totus infremuit, ac tantos ulutatus cum horridis clamoribus ejulando emisit, quod astantibus maximos terrores incussit. Et dum his fere per dimidiam horam bacchando institisset, tandem, ut Deo placuit, vas, quod diu possederat, reliquit. Mulier cum se esse liberatam sensit, manus astantibus porrexit, ut eam erigerent, quia vires non habuit. Tunc ante principale altare sancti Nycholai se prostravit, etc.

18. Ibid., p. 63 : Nam demon litteris vestris, quas, Spiritu sancto dictante, misistis, conjuratus, vas possessum per brevem horam reliquerat : sed heu! nescimus quo judicio Dei, rediit, vasque derelictum denuo invadens, illud nunc acrius quam prius fatigat.

19. Ibid., p. 65 : Vade, Sathanas, de tabernaculo corporis mulieris; et da in eo locum Spiritui sancto.

20. Ibid. 
étymologie faisait dériver anus, autre synonyme de vetula, d'un « a » privatif et du «nous » grec, associant ainsi étroitement vieille femme et privation de raisonnement ${ }^{21}$. La vetula, qui concentrait en elle trois états méprisés ou fuis - féminité, vieillesse et simplicitas - se trouvait de fait à la pliure de deux domaines propices à la misogynie, la médecine et la théologie, étroitement imbriqués dans la pratique thaumaturgique particulière de l'exorcisme. Deux domaines où le savoir des femmes était particulièrement mal ressenti, car jugé dangereux en raison de son inconsistance scientifique même : la vetula guérisseuse aux connaissances empiriques pouvait prétendre tenir sa science d'une illumination divine et attirer de la sorte «à grand renfort d'eau bénite et de prières une foule de malades ${ }^{22}$. En désignant ainsi Hildegarde, le démon se fait donc l'écho d'une conception de la malignité de la femme portée à son comble par la figure de la vieille femme, symbole d'une double déviance, celle de la foi et celle du savoir ${ }^{23}$.

\section{Sobriquet et invective}

La féminité de la vetula était pure négativité ${ }^{24}$ (l'arrêt des menstruations la faisait passer pour particulièrement toxique, nulle purge ne venant plus évacuer son sang mauvais, et sa peau ridée, loin de la rendre inoffensive car désormais dépourvue d'attraits, reflétait la mort qu'elle portait en elle), et le sobriquet forgé par l'esprit malin pour stigmatiser les rides de Hildegarde reprend le même thème dans l'intention d'en faire rire. A corps déformé au point d'en être grotesque, nom déformé censé produire un effet comique : nomen ejus evertens, deridens, le texte est très clair en ce sens et un vocabulaire semblable est employé dans la Vita sancti Bernardi où le diabolus est qualifié à plusieurs reprises d'irrisor. Dans un cas comme dans l'autre, l'esprit mauvais se rend coupable de derisio, un de ces «péchés de langue » fustigés par les clercs du Moyen Age : la derisio, et ses synonymes dans le cadre des discours sur ces péchés (irrisio, subsannatio ou encore illusio), était résolument rejetée du côté du mal, comme contraire à la charité et intentionnellement méchante, la seule irrisio tolérée étant celle de Dieu manifestant son juste mépris envers ses ennemis ${ }^{25}$. Aussi le désir qu'a Bernard de rendre en quelque sorte au démon la monnaie de sa pièce en se moquant à son tour du moqueur est-il légitime, ce qui autorise son

21. J. AGrimi, C. CRISCIANI, «Savoir médical et anthropologie religieuse. Les représentations et les fonctions de la vetula $\left(X_{I I I}{ }^{e}-X^{e}\right.$ siècle) », Annales ESC, 1993, n 5, pp. 1281-1308, p. 1293.

22. Ibid., p. 1287.

23. Ibid., p. 1304.

24. Sur le motif de la vieille femme dans la satire, voir aussi J. Horowirz, S. MENACHE, L'humour en chaire. Le rire dans l'Église médiévale, Genève, Labor et Fides, 1994, chap. V: "La femme médiévale au miroir déformant de l'humour", pp. 187-242, notamment pp. 197198.

25. Cf. C. Casagrande, S. Vecchio, Les péchés de la langue, Paris, Le Cerf, 1991 pour la trad. frse. 


\section{LE RIRE}

hagiographe à associer au saint le même verbe qu'au diable quelques lignes plus haut (irridens servum Dei) et à écrire : Vir Dei... irrisorem irrisit ${ }^{26}$.

Il n'en demeure pas moins que Bernard est lui aussi ridiculisé en deux temps : c'est d'abord son comportement alimentaire qui doit susciter le rire à ses dépens, et Bernard, dont on connaît les critiques contre la gourmandise des moines, notamment par son Apologia ad Guillelmum, se voit qualifié de «mangeur de poireaux » et d' «avaleur de choux », porros edens et brassicas devorans ${ }^{27}$, comme si le démon, en se moquant de la diète de l'abbé, voulait insinuer qu'elle le privait certainement des forces physiques nécessaires à son expulsion - la possession était de fait le seul cas, note Pierre-André Sigal, où le malade était frappé de coups «s'adressant évidemment au démon qui avait pris possession du corps du malheureux $»^{28}$. Effectivement l'esprit continue de se moquer de Bernard en s'en prenant cette fois à son nom, et c'est le thème de l'insuffisance physique qui semble commander l'emploi des sobriquets Bernardulus et Syrulus, « Bernardinet » et «Sirule »: le saint précédemment invoqué en vain et celui qui veut le relayer sont logés à la même enseigne, et l'apparition conjointe des deux diminutifs traduit la volonté qu'a le diable d'amoindrir, de diminuer ses adversaires. Or on sait par ailleurs que Bernard était de constitution fragile et en mauvaise santé, souffrant notamment de l'estomac en raison de son mode de vie trop austère ${ }^{29}$. Outre que nos deux extraits confirmeraient, s'il en était besoin, que le corps est le lieu privilégié de l'injure ${ }^{30}$, on y trouve à l'œuvre un cratylisme de la moquerie et de l'invective: l'emploi de l'hypocoristique a pour objet d'induire l'idée d'une réelle petitesse de la personne à laquelle il s'applique. De même, dans le roman courtois, des noms pourvus de suffixes diminutifs pouvaient exprimer la faible importance sociale de ceux qui les portaient - comme le dit Philippe Ménard à propos des noms des bergers dans Aucassin et Nicolette, "A gent menue noms menus ! ${ }^{31}$ — et plus généralement, dans la vie courante, l'emploi de diminutifs pouvait passer pour une injure amenuisant l'honneur de la personne ${ }^{32}$.

On connaît l'importance du nom dans la culture médiévale et la conscience aiguë qu'on avait de sa signification, dans la réalité comme dans les textes. Les Étymologies d'Isidore de Séville en sont sans doute le

26. Vita sancti Bernardi, op. cit., col. 280.

27. Ibid.

28. P.-A. Sigal, L'homme et le miracle, op. cit., p. 29.

29. Sur le portrait physique de Bernard, voir J.-C. SCHMITT, « Saint Bernard et son image », dans Bernard de Clairvaux. Histoire, mentalités, spiritualité. Colloque de Lyon-Citeaux-Dijon, op. cit., pp. 639-657, pp. 644-646.

30. Voir à ce sujet L'invective au Moyen Age. France, Espagne, Italie, Atalaya, Revue française d'Études médiévales hispaniques, $\mathrm{n}^{\circ} 5$, automne-hiver 1994, Paris, Presses de la Sorbonne Nouvelle, 1995, par exemple M. MADERO, « L'injure et le corps en Castille aux XIII et XIV ${ }^{\mathrm{e}}$ siècles », pp. 231-246 et la conclusion, par Claude Gauvard, pp. 249-258.

31. P. MENARD, Le rire et le sourire dans le roman courtois en France au Moyen Age (1150-1250), Genève, Droz, 1969, p. 578.

32. C. Gauvard, "De grace especial ". Crime, État et société en France à la fin du Moyen Age, Paris, Publications de la Sorbonne, 1991, 2 vols, t. 2, p. 732. 
monument le plus impressionnant et son influence fut considérable : l'équation nomen = omen dura tout le Moyen Age et l'étymologie devint une véritable forme de pensée. Il ne faut bien sûr pas perdre de vue que l'œuvre d'Isidore est une gigantesque synthèse de l'héritage antique et de la culture chrétienne, et que, bien avant lui, l'idée selon laquelle le nom dit l'être a traversé la philosophie grecque et les textes fondateurs du christianisme - un des exemples les plus significatifs dans ce dernier domaine étant peut-être le deuxième baptême de l'apôtre Simon-Pierre par le Christ au moyen d'un jeu de mots célèbre. Comme le rappelle Ernst Robert Curtius, " les chrétiens étaient autorisés à interpréter les noms par Matthieu, XVI, 18, et par les innombrables explications de noms de l'Ancien Testament », et on justifiait par exemple le prénom de saint Paul, l'apôtre des Gentils, Paulus en latin, en alléguant qu'il était minimus apostolorum, « le plus petit des apôtres ${ }^{33}$.

$\mathrm{Au}$ Moyen Age cette importance donnée au sens des prénoms semble avoir trouvé son expression la plus exacerbée dans la Légende dorée composée par Jacques de Voragine au $13^{\mathrm{e}}$ siècle et qui devint par la suite une sorte de best-seller dans le domaine hagiographique. L'auteur y a compilé les Vies de très nombreux saints, et chaque notice est précédée d'un paragraphe explicitant l'origine et le sens du nom du saint. Son destin apparaît inscrit dans son nom et celui de Bernard de Clairvaux est ainsi commenté :

Bernard vient de ber, puits, fontaine, et de nard, plante d'après la glose sur le Cantique des cantiques. Humble, d'une nature échauffante et odoriférante. En effet saint Bernard fut échauffé d'un fervent amour; il fut humble dans ses habitudes et odoriférant par la suavité de sa réputation ${ }^{34}$.

En fait, «Bernard» se décompose en bern-hardo, berin hard, «ours fort ${ }^{35}$. Jacques de Voragine prend évidemment avec l'étymologie des libertés nécessaires à son propos et met les mots mêmes au service de l'hagiographie, mais est-ce à dire que la signification originelle d'un prénom, aussi usité fût-il que "Bernard", un des 23 noms les plus courants chez les clercs de Bourgogne d'après les travaux de Patrice Beck ${ }^{36}$, échappait à ceux qui le portaient ou l'utilisaient? Il semble que non, et plusieurs exemples montrent qu'un nom d'origine tudesque restait chargé de sens, à l'époque qui nous intéresse.

Tous n'avaient évidemment pas la même aisance avec le langage que Hrotsvita de Gandersheim, la savante chanoinesse saxonne du $10^{\mathrm{e}}$ siècle : en se présentant dans son œuvre par la formule Ego, clamor validus

33. Cf. E. R. Curtius, La littérature européenne et le Moyen Age latin, trad. J. Brejoux, Paris, PUF, 1956, pp. 601-603.

34. JaCQues de VoRagine, La légende dorée, trad. J.-B. M. Roze, Paris, Flammarion, 1967, 2 vols, t. 2, pp. 111-112.

35. R. DFIORT, Introduction aux sciences auxiliaires de l'histoire, Paris, Armand Colin, 1969, p. 220.

36. Cf. P. BECK, "Anthroponymie et désignation des clercs en Bourgogne ", dans Genèse médiévale de l'anthroponymie moderne, M. Bourin éd., ${ }^{\mathrm{re}}$ et $\mathrm{II}^{\mathrm{c}}$ journées d'Azay le Ferron, Tours, Publications de l'Université de Tours, 1992, t. II-1, pp. 99-104. 


\section{LE RIRE}

Gandesheimensis, elle soumit à la postérité une énigme qui ne fut résolue qu'au début du siècle dernier par Jacob Grimm. En réalité, elle ne faisait que latiniser les deux termes de vieux saxon dont était formé son prénom, hruot (hrôt ou hrodh, Ruf en allemand moderne = clamor) et sui(n)d (swîth ou $s w \hat{\imath} d h=$ validus, ayant donné l'allemand geschwind $)^{37}$. Mais des esprits moins brillants se montraient capables de restituer un sens dans les prénoms, tel le biographe de Godelive, la mal mariée chère à Georges Duby, traduisant son nom par cara $\operatorname{Deo}^{38}$, ou Élisabeth de Schönau, jeune mystique émule de Hildegarde lui disant combien elle la jugeait digne du prénom qu'elle portait $^{39}$.

Élisabeth à vrai dire se trompait quelque peu au regard de nos connaissances actuelles (Hild signifie « combat», et gard, « demeure ») ${ }^{40}$, comme ce troubadour étudié par Martin Aurell glosant le prénom «Frédéric » par «cela veut dire " frein des puissants" » paix », «Fried-reich». Mais ce qui compte c'est que chacun, même les moins instruits comme en témoignent certaines injures étudiées par Claude Gauvard ${ }^{42}$, disposait d'un système explicatif pour l'univers de prénoms dans lequel il se mouvait, y compris pour le sien propre, et que partant, toute atteinte au nom en prenait d'autant plus d'impact. Saint Bernard sentait-il que son prénom l'apparentait à un ours dans ses forces ? Plusieurs éléments invitent à le supposer : la grande sensibilité au langage que nous lui connaissons d'une part, et qui lui valut le surnom de doctor mellifluus, et d'autre part la nette conscience du sens de son propre nom que devait avoir, d'après la lettre d'Élisabeth de Schönau, sa contemporaine Hildegarde, et que l'on extrapolerait volontiers à l'abbé de Clairvaux ${ }^{43}$. Quoi qu'il en soit, les sobriquets dont se voient affublés les deux saints par leur adversaire font

37. Cf. M. Goullet, Les drames de Hrotsvita de Gandersheim, édition critique avec introduction, traduction et notes, thèse de doctorat dactylographiée, Université de Metz, Faculté des Lettres, Section de latin, 1993, p. 7. Je remercie Monique Goullet de m'avoir donné un exemplaire de sa thèse.

38. G. DuBy, Mâle Moyen Age : de l'amour et autres essais, Paris, Flammarion, 1988, p. 61.

39. "Tu sei veramente quello che dice il tuo nome (cioè secondo un etimologia, solo in parte esatta, stimolo della fortezza) perchè il pungolo di Dio opera in te con fortezza mirabile nell'edificazione della sua Chiesa », cité et traduit par R. MANSELLI, « Amicizia spirituale ed azione pastorale nella Germania del secolo XII : Ildegarda di Bingen, Elisabetta ed Ecberto di Schoenau contro l'eresia catara ", dans Studi in onore di Alberto Pincherle. Studi e materiali di storia delle religioni, Rome, Edizioni dell'Ateneo, 1967, 2 vols, t. 1, pp. 302-313, pp. 307308.

40. R. DilorT, Introduction aux sciences auxiliaires de l'histoire, op. cit., p. 220.

41. qe Fredericx / volt aitan dir com fres de ricx; $\mathrm{cf}$. M. AuRELl, La vielle et l'épée : troubadours et politique en Provence au XIII siècle, Paris, Aubier, 1989, p. 338, n. 99.

42. Voir par exemple " De grace especial »..., op. cit., p. 733 : « Perenelle Sagette, une forte mégère, s'en prend à son voisin, un nommé Chardebeuf, en jouant sur son nom : "Chardebeuf! Ribaut, ruffien, maquereau, vendeur de char vive aux moines!" ".

43. Bernard lui-même se montre capable de jouer sur le nom de l'adversaire : dans une lettre à Innocent III, il moque ainsi Abélard et l'antipape Pierre Léon, en comparant le premier à un dragon, le second à un lion, « au prix d'un jeu de mots facile»; cf. A. DimIER, « Outrances et roueries de saint Bernard », dans Pierre Abélard. Pierre le Vénérable. Les courants philosophiques, littéraires et artistiques en Occident au milieu du XIT siècle, Colloque de l'abbaye de Cluny 2-9 juillet 1962, Paris, CNRS, 1975, pp. 655-670, p. 659. 
remarquablement mouche : un homme censé de par son prénom être fort comme un ours se fait ni plus ni moins traiter de «minus », et une femme supposée avoir la solidité d'une forteresse n'est pas appelée autrement que « ruine », en d'autres termes, le démon, en jouant sur les noms de ses adversaires, s'attaque à leurs failles en leur jetant au visage la discordance entre leur nom et leur être. Comme l'écrit Claude Gauvard, «si l'invective pèse d'un poids aussi lourd », n'est-ce pas que la parole est capable « de créer un état $\gg^{44}$ ?

\section{Sérieux comme un saint}

Nous avons tenté jusqu'ici de démonter les mécanismes selon lesquels le rire démoniaque s'était exercé aux dépens des deux saints; reste à s'interroger sur la portée et la fonction de tels épisodes dans des textes hagiographiques, et à chercher dans quel but un biographe donnait à voir son « candidat » dans une situation aussi peu avantageuse. Pour cela, il faut nous pencher sur la conception du rire en vigueur dans l'Église du $12^{\mathrm{e}}$ siècle, mais aussi tenter de cerner l'idée que s'en faisaient personnellement Bernard et Hildegarde, à la lumière de leurs œuvres et de ce que nous connaissons de leur caractère.

Que savons-nous au juste de la personnalité de saint Bernard? Dom Leclercq a relancé la question, constatant que les Vitce de l'abbé de Clairvaux avaient récemment fait l'objet d'interprétations tendant «à réduire l'admiration qu'elles étaient supposées provoquer $»^{45}:$ le livre premier de la Vita prima excellerait par exemple «à transformer en autant de vertus ce qui n'était en Bernard que des tendances regrettables ». On peut en tout cas tenir pour acquis, avec Jacques Berlioz, son « caractère affirmé » et sa «volonté farouche de développer l'ordre cistercien $»^{46}$ : incontestablement intransigeant et austère, Bernard déchaîna plus d'une inimitié et fut la cible de nombreuses attaques, à la fois pour ses prises de position personnelles telle sa condamnation d'Abélard, et en tant que prestigieux représentant d'un ordre dont l'expansionnisme n'était pas du goût de tous. Il fit donc dès son vivant l'objet de violentes critiques dans différentes satires composées par des clercs, et cette littérature mit en doute jusqu'à ses pouvoirs guérisseurs, alors qu'il jouissait en ce domaine d'une "indéniable popularité $»^{47}$ : une "légende noire visant le thaumaturge ${ }^{48} s^{\prime}$ 'était développée et continua de fleurir après sa mort. Ainsi le Gallois Gautier Map (v. 11301209 ou 1210), dans ses Balivernes de courtisans, montre-t-il Bernard en

44. C. Gauvard, sa conclusion dans L'invective au Moyen Age. France, Espagne, Italie, op. cit., pp. 256-257.

45. Cf. J. LeClerCQ, «La "paternité" de saint Bernard et les débuts de l'ordre cistercien ", Revue bénédictine, 103, $\mathrm{n}^{\circ} 3-4,1993$, pp. 445-481, p. 452.

46. J. BeRLIOZ, "Saint Bernard dans la littérature satirique », loc. cit., p. 211.

47. Cf. P.-A. SigaL, L'homme et le miracle..., op. cit., p. 18: «Lors de son voyage en Germanie en 1146-1147, les malades ne cessaient de se présenter le long des routes qu'il empruntait ».

48. J. BeRLIOZ, «Saint Bernard dans la littérature satirique », loc. cit., p. 222. 
situation d'échec dans trois tentatives de miracles, dont un de guérison d'un possédé : à peine libéré de son démon, le possédé ne trouva rien de mieux pour remercier le saint que d' "envoyer de toutes ses forces des pierres sur l'abbé, le poursuivant avec insistance comme il s'enfuyait par les chemins aussi longtemps qu'il le put ». L'action est située à Montpellier, ville où apparemment Bernard ne s'est jamais rendu, mais si le récit reste vraisemblable c'est que «son auteur s'est appuyé sur les passages des Vies du saint où on le voit aux prises avec des possédés qui - mais avant leur guérison - lui donnent des coups ou l'injurient violemment ${ }^{49}$. L'épisode qui nous occupe est un de ces passages susceptibles d'avoir inspiré Gautier Map, et on saisit ici toute l'ambiguïté d'un tel texte : son auteur a-t-il réellement adapté au but assigné à la Vie de saint Bernard les informations dont il disposait, notamment sur l'activité thaumaturgique du saint ? Ou, à force de détails dans ses récits d'exorcismes - pour lesquels il semblait avoir une "prédilection remarquable $»^{50}$ - n'y a-t-il pas introduit aussi les armes de leur propre contradiction? Présenter Bernard aux prises avec des possédées qui, à Milan comme à Pavie, l'injuriaient, le frappaient et en tout cas le tenaient en échec, c'était courir le risque que le texte, loin de forcer l'adhésion et l'admiration, suscite une réaction inverse : l'incrédulité, voire la critique ouverte et, en définitive, le refus de reconnaître la sainteté du personnage que toute la Vita, pourtant, tendait à démontrer.

A vrai dire, les auteurs des satires accablant Bernard pouvaient-ils trouver un meilleur exutoire à leur hostilité que dans ce rire libérateur aux dépens d'un saint connu pour son sérieux inébranlable? «Saint Benoît définissait le dixième degré d'humilité du moine par le refus de se laisser aller facilement au rire, et le onzième degré par une parole douce, humble, sérieuse, rare, raisonnable, sans clameur et sans rire $»^{51}$ : brisant la loi du silence, le rire semblait incompatible avec une vie de componction et de pénitence, et certainement, Bernard vécut en conformité avec ce précepte, comme on peut en juger entre autres par cet extrait de la Légende dorée, une fois faite la part de l'intention apologétique de l'auteur:

S'il riait, c'était toujours de telle sorte qu'il lui fallait faire des efforts pour rire plutôt que pour réprimer des ris : il fallait qu'il les excitât plutôt qu'il ne les retînt. Comme il avait coutume de dire qu'il y avait trois genres de patience, savoir, 1) patience pour les paroles injurieuses, 2) patience pour le dommage dans les biens, et 3) patience dans les maladies du corps, il prouva qu'il les possédait tous par les exemples qui suivent $[\ldots]$.

Outre les portraits qu'ont laissés de lui des auteurs contemporains ou postérieurs, les propres écrits de Bernard (et ceux qui lui sont attribués) nous renseignent sur sa conception du rire. Le refus du rire est une vertu

49. Ibid., pp. 225-226.

50. A. H. BREDERo, "La vie et la Vita prima», loc. cit., p. 71.

51. J. LE GoFF, "Le rire dans les règles monastiques", dans Haut Moyen Age, culture, éducation et société. Études offertes à Pierre Riché, M. Sot coordonnateur, Nanterre, Publidix, 1990, pp. 93-103, p. 99. 
distinguant les chrétiens des païens car seul le chrétien connaît la raison pour laquelle il ne faut pas rire, mais au contraire pleurer, à savoir la peur du Jugement dernier, comme le rappelle par exemple le Tractatus de ordine vitce en s'appuyant sur l'Évangile de Luc ${ }^{52}$. "Ta conversation sera sérieuse ou ne sera pas », dit-il en substance dans sa Formula honestae vita ${ }^{53}$, pour enjoindre ailleurs au lecteur de fuir toujours les ricanements, et de n'user du rire que rarement ${ }^{54}$. Ne compare-t-il pas le moine rempli de pensées vaines et secoué d'éclats de rire à une vessie gonflée de vent et percée d'un trou étroit obligeant ce vent à rendre des sons répétés, faute de pouvoir se libérer d'un seul coup 55 ? Sans être aussi haïssable que son pôle négatif, la cachinnatio à connotation diabolique, le rire est fortement suspect et s'inscrit à plein dans un mouvement plus général analysé en détail par Jacques Le Goff : c'est certes « le rire accompagné de ricanements et de tressautements ( subsannatio, risus cum cacchinis), le rire excessif, le rire briseur de silence, le rire grivois, qui est condamné sans appel mais la tendance à la prohibition du rire est nette ${ }^{56}$.

Hildegarde aussi en fait le propre du diable en différents endroits de son œuvre, à l'instar par exemple de son contemporain Rupert de Deutz, pour qui seul le diable rit, lorsqu'il parvient à abuser les croyants ${ }^{57}$ : elle fait volontiers parler les démons sur un registre inférieur ou comique dans ses poésies ou dans son drame liturgique Ordo virtutum, et dénonce dans un de ses chants dédiés à sainte Ursule le caractère dévastateur du rire diabolique ${ }^{58}$. Elle-même n'eut-elle pas à souffrir les moqueries des démons quand elle tomba malade après la libération de Sigewize, comme le rapporte un passage autobiographique de sa Vita $^{59}$ ? Et quand la contestation avait couvé au Rupertsberg, où certaines sœurs trouvaient insupportable la forme de vie monastique que voulait leur imposer l'abbesse, c'est bien aux esprits malins qu'elle avait attribué les calomnies et moqueries auxquelles était en butte son sérieux. Mais surtout, avant même d'entreprendre de libérer la jeune obsessa qui nous retient, elle avait eu une vision lui montrant la foule

52. Cf. Tractatus de ordine vitce et morum institutione, PL 184, col. 568A : "Va vobis qui ridetis, quia flebitis vos ", Dominus ait (Luc VI, 25). Nos ridendi materiam inquirimus, ut hic ridentes, illic fleamus?

53. Formula honesta vita, PL 184, col. 1172A.

54. Cf. Varia et brevia documenta pie seu religiose vivendi, PL 184, col. 1173D : A cachinnis semper abstineas: rideas vero raro.

55. Liber de gradibus humilitatis et superbice; cité par J.-C. SCHMITT, La raison des gestes dans l'Occident médiéval, Paris, Gallimard, 1990, p. 153.

56. J. LE GOFF, « Le rire dans les règles monastiques », loc. cit., p. 101.

57. Cf. RuPERT De DeUTz, In Habacuc prophetam commentaria, 1, PL 168, col. 599 ss, cité par I. M. RIESNICK, "Risus monasticus. Laughter and Medieval Monastic Culture », Revue bénédictine, 97, 1987, pp. 90-100, p. 99.

58. Cf. Hildegarde DE Bingen, Louanges, prés. et trad. L. Moulinier, Paris, La Différence, 1990 , p. 82 : Sed Diabolus in invidia sua / istud irrisit, / qua nullum opus Dei / intactum dimisit ( le Diable, dans son envie, s’en moqua, car il n’a laissé intacte aucune œuvre de Dieu »).

59. Vita sancta Hildegardis, op. cit., p. 66 : Et vidi, quod nequam spiritus inde irridebant, cachinnando dicentes: "Wach! Ista morietur, et amici ejus flebunt, cum quibus nos confundit". 


\section{LE RIRE}

des anges déchus, et c'est sur cette armée d'esprits malins, qui ont contesté la puissance de Dieu dès leur création, que Hildegarde faisait peser la responsabilité du premier péché de dérision, error irrisionis ${ }^{60}$.

\section{Le rire, propre de l'homme?}

Or Hildegarde était aussi versée dans la médecine, et ses écrits reflètent une vision biologiquement orientée de la Création; le rire ne fait pas exception et le traité Causa et curae qui lui est attribué donne de ce phénomène une explication mêlant théologie et approche scientifique. Elle distingue deux types de joie, honesta laetitia et inepta latitia, et réfléchit également aux mécanismes et aux effets du phénomène contraire, la tristesse. L'inepta latitia, la « folle réjouissance » que Bernard liait au troisième degré de l'orgueil ${ }^{61}$, et le rire qui lui est associé ont ici un rapport étroit avec le plaisir charnel : le vent (terme dans lequel il faut peut-être voir une trace de la théorie galénique des pneuma) qui déchaîne le rire en l'homme en secouant son entrejambe et ses viscères est le même que celui qui provoque l'éjaculation, et les larmes qui souvent accompagnent le rire jaillissent des yeux comme la semence est éjectée quand le sang est secoué par l'ardeur du plaisir ${ }^{62}$. Or le plaisir découlant de l'union de l'homme et de la femme a changé de nature avec la Chute ; le péché originel a en effet eu des conséquences à tous les niveaux de la Création, notamment sur la constitution physique de l'homme et de certains animaux : il a provoqué, comme le dira aussi l'encyclopédiste Alexandre Neckam, la transformation des fluides dans l'organisme, d'où l'apparition d'une humeur noire, la mélancolie, phénomène qui trouva son pendant dans le règne animal avec la formation du venin des reptiles.

De même, avant la Chute, rire et ricanement n'existaient pas : ils sont le résultat de la dégradation de la "voix des joies suprêmes", vox superiorum gaudiorum, dont jouissait Adam lorsqu'il fut créé ${ }^{63}$, et une description du cheminement du rire à l'intérieur de l'homme donne la traduction biologique de cette assertion : lorsque le cœur de l'homme s'ouvre à la joie comme une fleur à la chaleur du soleil, cette joie va se loger dans le foie, à l'instar des aliments dans l'estomac. Un vent soufflant de ses moelles à la faveur de cette joie gagne l'entrejambe et de là, la rate, dont il envahit

60. Ibid., p. 57 : Quis est iste, qui tantam habet potestatem super nos? Hoc in invidia, odio et irrisione dicentes, adhuc in his perseverant, et omnia in his faciunt, quia errorem irrisionis primi incoeperunt.

61. J.-C. SchmitT, La raison des gestes dans l'Occident médiéval, op. cit., p. 153.

62. Cf. Hildegardis Causa et cura, P. KAISER éd., Leipzig, Teubner, 1903, p. 148 : Inepta enim loctitia et risus velut quandam societatem ad delectationem carnis habent, et ideo ventus ille, qui risum suscitat, de medulla hominis exiens femur eius et interiora concutit. Ac interdum prae nimia concussione risus aquam lacrimarum de sanguine venarum oculis hoc modo educit, quemadmodum etiam spuma seminis hominis aliquando de sanguine venarum per ardorem delectationis excutitur.

63. Ibid. : Nam sicut in provaricatione Ada sancta et casta natura prolem gignendi in alium modum delectationis carnis mutata est, ita etiam et vox superiorum gaudiorum, quam idem Adam habebat, in contrarium modum risus et cachinnorum versa est. 
peu à peu les veines, puis il gagne le cœur et emplit le foie, ce qui provoque en l'homme le rire et l'émission de sons semblables à des bêlements ${ }^{64}$.

Le rire mauvais ne l'est donc pas seulement en raison de sa lointaine origine, l'action du Diable : il est néfaste puisqu'en lui faisant produire des sons privés de sens, il ravale l'homme, à l'origine doté d'une voix céleste, au rang du bétail; en outre il nuit à sa santé car entre autres effets, il "blesse sa rate, met à mal son foie et crée un bouleversement total de ses humeurs ». Or la maladie naît, selon les conceptions médicales de l'époque, d'une rupture de l'équilibre des quatre humeurs qui composent le corps de l'homme (sang, bile, flegme et atrabile) ; aussi le Causa et curce fournitil, outre l'explication physique du phénomène, un remède contre le mal : le rire immodéré, le fou rire qui dessèche les poumons et secoue violemment le foie et les humeurs, peut être vaincu grâce à une préparation à base de noix de muscade, de sucre et de vin chaud ${ }^{65}$.

Ce traité proposant une double analyse du rire fait figure de pionnier dans l'Occident médiéval. Auparavant, bien sûr, différents auteurs s'étaient penchés sur ce problème qu'Aristote avait soulevé le premier en constatant que l'homme était le seul animal capable de rire. La question de savoir comment le rire propre à l'homme le positionnait par rapport à Dieu et au Diable, occupa de nombreux penseurs chrétiens et ce thème fut souvent abordé car il en allait de la recherche de l'essence même de l'homme: selon Helen Adolf, "homo animal risibile capax " était une définition possible, "homo animal mortale rationale » en était une autre ; la première distinguait l'homme des animaux, la seconde, de Dieu, et si le rire donnait tant à penser c'est qu'il pouvait être considéré comme la conséquence naturelle de la combinaison, en l'homme, de deux qualités aussi contradictoires que la mortalité et la raison ${ }^{66}$. Mais personne en Occident n'avait jusqu'alors reposé la question dans une perspective naturaliste comme l'avait fait Aristote, qui était arrivé à la conclusion que le siège du rire se situait dans le diaphragme ${ }^{67}$, ce que semble retrouver Hildegarde en assi-

64. Ibid., p. 149 : Cum enim scientia anima hominis nihil tristitia et nihil adversi et nihil mali in homine sentit, tunc cor ejusdem hominis ad latitiam se aperit, ut flores ad calorem solis se aperiunt, et mox iecur eamdem latitiam recipit ac eam in se retinet, ut stomachus cibum in se continet. Et cum sic homo aut de bonis aut de malis, quac sibi placent, latatur, tunc pradictus ventus interdum ex medulla exiens femur illius primum tangit et ita splen occupat atque venas ejusdem splenis implet et se ad cor extendit et iecur replet ita quod hominem ridere facit atque vocem ejus similem voci pecorum in cachinnis educit.

65. Ibid., p. 199 : Qui multu et immoderato risu motus et concussus dolet, nucem muscatam pulverizet et huic bis minus zaccari addat et sic calefacto vino imponat et illud tam jejunus quam pransus bibat. Immoderatus risus enim pulmonem arefacit et iecur concutit, sed calor nucis muscatce iecur sanat, et calor et succus zaccari pulmonem renovat. Et cum hac alterato calore vini temperatur et ita sumuntur, bonos humores per immoderatum risum destitutos restituunt.

66. Cf. H. Adolf, « On Medieval Laughter », Speculum, 22, 1947, pp. 251-253, p. 253.

67. Cf. Aristote, Les parties des animaux, III, $10,672 \mathrm{~b}$, trad. P. Louis, Paris, Les Belles Lettres, 1956, p. 96 : «Ce qui prouve que, quand il reçoit de la chaleur, le diaphragme manifeste aussitôt qu'il éprouve une sensation, c'est aussi ce qui se passe dans le rire ». Voir aussi ses Problèmes, trad. P. Louis, Paris, Les Belles Lettres, 1994, p. 87 : «Et c'est pourquoi les gens 


\section{LE RIRE}

gnant l'origine du rire dans le jecur: lorsqu'en effet un auteur comme Aelred de Rielvaux, partisan d'un usage très modéré de la parole dans la vie monastique ${ }^{68}$ et farouche ennemi du rire, évoque la diffusion de ce «venin bu avec délice» dans « les viscères et dans les membres », le vocabulaire anatomique est là pour donner plus de force à sa condamnation du rire, mais nul souci de réalisme ne l'anime ${ }^{69}$.

La complexité du rire tient à sa double nature, à l'intersection de l'âme et du corps, du physique et du mental, et le Causa et curce, qui expose le phénomène à la fois en tant qu'affection de l'âme et dans sa mécanicité de phénomène physique sans exclure pour autant la perspective théologique, n'a apparemment pas d'équivalent. Même Avicenne, dont le De Anima, rédigé au début du $11^{\mathrm{e}}$ siècle et traduit en latin entre 1152 et 1166 , pénétra très tôt en Occident, traite le rire comme une des passions de l'âme, sans décrire la naissance et la progression de ce phénomène dans le $\operatorname{corps}^{70}$ : se situant résolument dans la ligne du traité $D e$ l'Ame, il ne retient que l'aspect intellectuel de la leçon aristotélicienne, «Le rire est une espèce de dérangement et de surprise $\gg^{71}$, et l'arrière-plan théologique est absent de son exposé. D'un autre côté, la littérature strictement médicale, comme les traités salernitains dont Hildegarde a manifestement eu connaissance ${ }^{72}$, aborde la question du rire et des larmes à la lumière de la théorie des humeurs et de la doctrine des tempéraments. Selon l'humeur qui dominait en eux, les êtres humains étaient en effet distribués en quatre catégories, mélancoliques, flegmatiques, sanguins et colériques: la complexion la plus encline à l'hilarité était le tempérament sanguin, tandis que la disposition à tristesse, depuis Hippocrate, caractérisait les mélancoliques ${ }^{73}$. En

frappés au diaphragme éclatent de rire. Car ce n'est pas en n'importe quel endroit que se déclenche le rire ».

68. Aelred De Rielvaux, De vita eremitica, PL 32, col. 1454, cap. VII : Raro loquatur, id est certis et constitutis horis [...]. Quomodo loquatur, id est humiliter, moderate, non alta voce, nec dura blanda, nec mixta risu. Nam si hoc ad quemlibet virum honestum non pertinet, quanto magis ad feminam?

69. Ibid., col. 1452, cap. III : Os interea in risus cachinnosque dissolvitur, et venenum cum suavitate bibitum per viscera membraque diffunditur.

70. Cf. Avicenna latinus, Liber de Anima seu Sextus de Naturalibus, Pars V, cap. I, édition critique de la traduction latine médiévale par S. VAN RIET, Louvain-Leyde, E. Peeters-J. Brill, 1972, 2 vols, t. 2, pp. 73-74: De proprietatibus autem hominis est ut, cum apprehenderit aliquid quod rarissimum est, sequitur passio quar vocatur admiratio, quam sequitur risus, sed cum apprehenderit aliquid quod est noxium, sequitur passio qua vocatur anxietas, quam sequitur luctus.

71. Aristote, Problèmes, op. cit., p. 87.

72. Sur la question des sources des écrits scientifiques de Hildegarde, je me permets de renvoyer au chapitre VII, "Sources et influences", de l'ouvrage que j'ai tiré de ma thèse, Le manuscrit perdu à Strasbourg. Enquête sur l'œuvre scientifique de Hildegarde, Paris, Publications de la Sorbonne-Presses Universitaires de Vincennes, 1995. Les Questions salernitaires éditées par B. LAWN, avec lesquelles l'œuvre de Hildegarde offre des points de convergence, évoquent aussi le rire, ses rapports avec le spleen, et ses causes (cf. B. LAWN, The Prose Salernitan Questions, Londres, 1979, pp. 88, 95, 138 et 179).

73. Jackie Pigeaud fait toutefois remarquer qu'un auteur médiéval comme Paul d'Égine a mis le rire au nombre des signes de la mélancolie ; $\mathrm{cf}$. J. PIGEAUD, La maladie de l'âme. Etudes 
témoignent entre autres ces extraits du Regimen sanitatis salernitanum, régime de santé ayant vu le jour dans le milieu médical salernitain et qui connut une grande diffusion ${ }^{74}$ :

De sanguineis :

Natura pingues isti sunt atque jocantes,

Semper rumores cupiunt audire frequentes,

Hos Venus et Bacchus delectant, fercula, risus,

Et facit hos hilares, et dulcia verba loquentes.

Omnibus hi studiis habiles sunt et magis apti :

Qualibet ex causa nec hos leviter movet ira.

Largus, amans, hilaris, ridens, rubeique coloris,

Cantans, carnosus, satis audax atque benignus ${ }^{75}$.

Le sanguin apparaît comme un amoureux de la vie sous toutes ses formes, auquel s'oppose, par sa totale inaptitude à la joie et au rire, le mélancolique tel qu'il ressort de ces lignes :

De melancholicis :

Restat adhuc tristis cholerae substantiae nigrae,

Quae reddit pravos, pertristes, pauca loquentes.

Hi vigilant studiis, nec mens est dedita somno:

Servant propositum : sibi nil reputant fore tutum.

Invidus et tristis, cupidus, dextraeque tenacis

Non expers fraudis, timidus, lutei coloris ${ }^{76}$.

Le Causa et curae se fait l'écho de ces théories et campe les différents tempéraments en une galerie de portraits dont l'originalité tient notamment à la présentation des caractères féminins et masculins en des cadres séparés. Le mélancolique y est bien un être pétri de tristesse et d'inquiétude et inapte à la joie, et si ce portrait est si négatif, c'est que l'humeur qui domine en lui est un des plus funestes effets de la Chute ${ }^{77}$ : ce qui est actuellement

sur la relation de l'âme et du corps dans la tradition médico-philosophique antique, Paris, Les belles Lettres, 1981, p. 463. Hildegarde a peut-être eu accès au De melancolia de Constantin.

74. Le noyau primitif, formé d'environ trois cents vers rassemblés et commentés au $13^{\mathrm{e}}$ siècle par Arnaud de Villeneuve, fut progressivement complété au fil des siècles, mais c'est évidemment au noyau d'origine, tenu par la critique pour le texte latin le plus authentique, que nous faisons ici référence.

75. Regola sanitaria salernitana. Regimen sanitatis salernitanum, version italienne de Fulvio Gherli, Salerne, Ente provinciale per il Turismo, 1966, p. 73.

76. Ibid., p. 77. Voir aussi p. 81, De abundantia melancholia: :

Humorum pleno dum fax in corpore regnat,

Nigra cutis, durus pulsus, tenuisque urina,

Sollicitudo, timor, tristitia, somnia tetra;

Acesunt ructus, sapor, et sputamine idem.

Levaque pracepue tinnit vel sibilat auris.

77. Hildegardis causae et curae, op. cit., p. 38 : Et hac melancolia nigra est et amara et omne malum efflat ac interdum etiam infirmitatem ad cerebrum et ad cor quasi venas ebullire facit atque tristitiam et dubietatem totius consolationis parat, ita quod homo nullum gaudium habere potest, quod ad supernitatem et ad consolationem prosentis vitoe pertinet. 


\section{LE RIRE}

humeur noire en l'homme luisait auparavant « comme du cristal » en Adam car quand tout éclat s'est éteint en lui, la mélancolie s'est formée par coagulation dans son sang, porteuse de tristesse et de désespérance comme la suggestion du diable ${ }^{78}$.

La tristesse déplaît fondamentalement à Hildegarde : elle est à la fois offense à la beauté de la Création, qui doit réjouir le véritable croyant, et mère de doute et de colère ${ }^{79}$. La tristesse a évidemment une traduction physique ${ }^{80}$ et le Causa et curae recommande d'en endiguer les effets grâce à quelques remèdes, comme la saignée également préconisée par le Regimen sanitatis salernitanum. Mais la condamnation du rire n'est somme toute pas plus vigoureuse que celle de la tristesse; gaudium et latitia, et leurs manifestations, sont dans tous les cas préférables à tristitia, ce qui est particulièrement sensible dans la distinction opérée entre larmes de joie et larmes de tristesse : seules les premières sont louables, et elles sont dites plus douces, leniores, que les secondes, car elles jaillissent sans dommage pour le corps. Gaudium et latitia suscitent des pleurs qui coulent « comme une source suave », sans « abîmer le cœur, sans dessécher le sang, ni meurtrir la chair ou obscurcir les yeux ${ }^{81}$. Autant de maux qui accompagnent en revanche les larmes dues à la tristesse, qui montent aux yeux «telles une fumée amère », et font autant de mal à l'homme que des «aliments mauvais »: l'eau de ces larmes ne jaillit-elle pas du sang de l'homme, «par l'effet des soupirs et des gémissements, de la même façon que son sperme sort de son sang et de ses moelles ${ }^{82}$ ? Aussi les larmes du pénitent qui regrette ses péchés, par exemple, ne sauraient-elles être que des pleurs pour ainsi dire mixtes ${ }^{83}$ : la joie doit s'y mêler, compensant ainsi les défauts des larmes dues à la seule tristesse.

Par l'analogie introduite entre larmes de tristesse et émission séminale, le rire et les larmes sont mis sur le même plan : de même qu'il y a deux types de joie, il y a une bonne et une mauvaise façon de pleurer. Le critère de jugement est le souci du corps, qui prime sur une dichotomie opposant traditionnellement le bien des larmes au mal du rire : les mauvaises larmes sont celles qui nuisent au corps, et le rire négatif est le rire excessif qui secoue l'organisme. Tout en faisant siens les soupçons pesant sur le rire à son époque, Hildegarde fait converger médecine et morale dans son approche de la vie affective et se singularise ainsi radicalement.

78. Ibid., p. 143 : quia cum splendor in eo extinctus est et melancolia in sanguine ejus coagulata est, de qua tristitia et desperatio in eo surrexerunt, quoniam diabolus in casu Adae melancoliam in ipso conflavit, qua hominem aliquando dubium et incredulum parat.

79. Ibid., p. 146 : post tristitiam vero ira exsurgit.

80. Ibid., par exemple p. 153 : Sed alii homines sunt, qui triste et marens cor sape habent, et eadem tristitia interiores venulas, quae sanguinem per corpus ferunt, constringit, ita quod aliqua eorum per modicum vulneratur. Unde et illa guttas sanguinis ad interiora paulatim fundit et ita homo ille aliquando sanguinem evomit [...].

81. Ibid., p. 148.

82. Ibid., p. 147.

83. Ibid., p. 148 : Si quis panitendo peccata sua deflet, ha lacrima tristitia et latitia permixtoe sunt et tantum oppressa mente sine fumo educuntur. 
Ces deux extraits ont permis de dégager un certain nombre de traits du rire au Moyen Age que corroborent d'autres sources ; en s'interrogeant sur les différentes attitudes, théoriques ou pratiques, coexistant alors par rapport à ce phénomène que l'on pouvait relier tant à la question du salut de l'âme qu'à celle de la santé du corps, on a pu saisir plus profondément leurs implications, dans toute leur complexité. Ainsi a-t-on pu toucher du doigt les problèmes liés à la présence d'un certain comique dans une Vie de saint : il n'était certes pas tout à fait étranger au genre hagiographique, comme l'a noté E. R. Curtius, s'il s'agissait de montrer grâce à un élément humoristique le triomphe des forces du bien sur des adversaires comme les païens, les méchants ou encore le diable, dont les saints parvenaient à se jouer ${ }^{84}$. Or dans les deux Vitce sur lesquelles nous nous sommes penchés, le diable a pour un temps le dessus, le comique s'exerce aux dépens des personnages dont il s'agit de prouver la sainteté, et cet emploi du rire au détriment de deux figures importantes du clergé régulier du milieu du $12^{\mathrm{e}}$ siècle a d'autant plus d'impact que la répression du rire fut un des soins constants des législateurs monastiques. De fait, dans un cas comme dans l'autre, le choix délibéré de donner à voir le saint dans une situation d'échec, aux prises avec le ridicule, pour le faire sortir grandi de sa lutte avec le démon, ne s'avère pas sans risque : la version donnée par Theodoricus de l'exorcisme, en soi fort peu orthodoxe, opéré par Hildegarde, ne servit peutêtre pas sa cause aux yeux de ceux qui eurent à se prononcer pour ou contre sa canonisation, et Arnaud de Bonneval, comme d'autres auteurs de Vitce de Bernard, fit en un sens le lit de ses détracteurs. Là où un auteur avait voulu montrer un martyr du rire en lutte contre le diable et en route vers la sainteté, il était en définitive loisible à son humain lecteur, par essence homo risibilis, «capable de rire », de ne voir qu'un objet de risée.

Laurence MOULINIER

Université de Poitiers

84. Cf. E. R. Curtius, La littérature européenne et le Moyen Age latin, op. cit., pp. 527528. 